\title{
Por el futuro de la nación. La formación de los profesores para adolescentes de las escuelas secundarias y preparatorias públicas en la Ciudad de México (1910-1934)
}

\author{
For the Future of the Nation. \\ Teacher Training for Adolescents in \\ Public Junior and Senior High \\ Schools in Mexico City (1910-1934)
}

\section{Ivonne Meza Huacuja}

Universidad Nacional Autónoma de México

ivy.meza@gmail.com

\section{Resumen}

El objetivo del presente artículo es analizar el proceso de profesionalización de los maestros de enseñanza secundaria a partir de la aparición de nuevas concepciones científicas sobre la adolescencia desde finales del siglo xıx y hasta principios de la década de 1930 en México. Por medio de la aplicación de tales concepciones se buscó la difusión y sobrevivencia de proyectos nacionales y modelos arquetípicos sobre los futuros hombres y mujeres que encabezarían e impulsarían el progreso del país. Por otro lado, uno de mis propósitos en las siguientes páginas es mostrar un primer acercamiento a la historia del establecimiento de instituciones educativas en la Ciudad de México, como fueron la Escuela Nacional de Altos Estudios y la Escuela Normal Superior, enfocadas en la formación de especialistas en el tratamiento de adolescentes. De forma breve, haré referencia a las transformaciones de los planes de estudio de las mencionadas escuelas basados en las supuestas necesidades de dicho grupo de edad. Mi atención se centrará en la cátedra de Psicología de la Adolescencia impartida por Ezequiel A. Chávez, en 1922.

Palabras clave: profesionalización docente, profesores de escuelas secundarias, adolescencia, educación, México. 


\section{Abstract}

The purpose of this article is to trace the process of professionalization of secondary school teachers in Mexico from the end of the nineteenth century to the early 1930s in relation to the international emergence of new scientific notions of adolescence. These new theories were implemented to enforce some archetypical models of men and women in the post-revolutionany era for the progress of the nation. This piece of research also shows the history of the new training courses for educators, the new curricula oriented to adolescent specific needs and the formation of new institutions, such as the Escuela Nacional de Altos Estudios and the Escuela Normal Superior. My focus will be on the Psychology of Adolescence chair taught by Ezequiel A. Chávez in 1922

Keywords: professionalization of teachers, high schools, adolescence, education, Mexico.

\section{Introducción}

Las últimas décadas del siglo XIX constituyeron un periodo de incorporación de las nociones modernas sobre la adolescencia y los adolescentes en el mundo occidental. Si bien el adolescente apareció en la literatura medieval y renacentista como un personaje tipo, fue a partir de los avances médicos decimonónicos y de la constitución de la psicología experimental que sus cambios fisiológicos y de conducta se convirtieron en objeto de interés y de preocupación para científicos, pedagogos y funcionarios. A grandes rasgos, entre los especialistas de la época la adolescencia fue considerada como la primera etapa de la juventud cuyo inicio, alrededor de los doce años, estaba determinado por la aparición de caracteres sexuales secundarios, por cambios drásticos en el comportamiento como el rápido enamoramiento, la rebeldía, la ensoñación, el instinto gregario, entre otros. ${ }^{1}$

La mayoría de las investigaciones científicas sobre la adolescencia se llevaron a cabo en Estados Unidos, en cuyas ciudades se percibía un aumento en la criminalidad, y sus resultados se aplicaron en las instituciones encargadas de la educación de los jóvenes. Mediante su implementación, generalmente en forma de planes de estudio, actividades escolares y contenidos educativos, se persiguió, además de un control más riguroso sobre un sector juvenil como el de los adolescentes, la formación de los futuros ciudadanos. Así pues, las políticas y métodos educativos encarnaron las preocupaciones y expectativas sobre un futuro cercano. La educación de los jóvenes significó la sobrevivencia de proyectos de nación y de grupos

\footnotetext{
${ }^{1}$ Para más información sobre la historia de las distintas caracterizaciones y conceptualizaciones sobre la adolescencia y los adolescentes en México, véase Meza Huacuja (2015)
} 
sociales (religiosos, políticos, de clase social y género) cuyos ideales se reflejaron en los planes de estudio y en las actividades extracurriculares.

En México, el abogado, funcionario, maestro y psicólogo Ezequiel A. Chávez (1868-1946) fue uno de los principales introductores de las teorías psicológicas y de las propuestas pedagógicas enfocadas en los adolescentes. Su labor al frente de instituciones educativas cardinales como la Escuela Nacional Preparatoria (ENP), la Escuela Nacional de Altos Estudios (ENAE) y la Universidad Nacional contribuyó enormemente al establecimiento de centros educativos enfocados en la formación de adolescentes, en la implementación de novedosas técnicas pedagógicas y en la capacitación de docentes especializados en el trato y tratamiento de los adolescentes.

Uno de los objetivos del presente trabajo es ofrecer un primer acercamiento a la historia de la formación de educadores especialistas en dicho grupo de edad, considerando teorías y discusiones que en ese momento se generaron entre funcionarios y pedagogos extranjeros y mexicanos. Algunas de ellas fueron utilizadas y difundidas por Ezequiel A. Chávez en su curso Psicología de la Adolescencia, impartido desde 1922, y en su libro Ensayo de psicología de la adolescencia (1928).

La importancia en el estudio de dicho sector social contribuye a entender, por lado, el interés de distintos sectores -en este caso particular me enfocaré en la Universidad Nacional (a través de la ENAE) y la Secretaría de Educación Pública- por incidir en el nuevo proyecto nacional posrevolucionario a partir de su control sobre la formación de los adolescentes, considerados los futuros ciudadanos del país, por medio de especialistas entrenados en su educación.

Cabe resaltar que si bien la preparación de docentes de enseñanza secundaria y preparatoria ha sido mencionada por algunos autores (Duncoing, 1990; Caño, 1996; Loyo, 1999; Sandoval Flores, 2000), no se ha explorado desde la perspectiva que propongo en este artículo. Así, aunque se han realizado trabajos exhaustivos sobre la historia de la ENAE de la Universidad Nacional y de sus planes de estudio, no pude localizar alguno que la vinculara con las reflexiones sobre la adolescencia en aquella época.

Para la presente investigación estimé importante hacer un repaso de la historia de la ENAE y del establecimiento de los cursos de especialización docente en dicha institución. Particularmente, me enfoqué en la cátedra de Psicología de la Adolescencia, impartida por Chávez desde 1922 e instituida como asignatura obligatoria para los futuros educadores de adolescentes.

Otro de los puntos que este artículo considera es la elaboración de un breve repaso de los programas de estudio y de las temáticas incluidas en las distintas materias. Por medio de dicho procedimiento pretendo delinear cuáles eran las expectativas sobre los formadores de adolescentes dentro de las instituciones de educación pública en un periodo temporal 
específico. La especialización de maestros de educación posprimaria (particularmente de las escuelas secundarias y preparatorias), su diferenciación de los docentes de enseñanza primaria, el interés en su preparación en áreas específicas (como en el desarrollo y características psicológicas de un grupo de edad particular, la implementación de castigos y la orientación sexual, entre otras) dan cuenta de las representaciones y expectativas de ciertos sectores adultos sobre los adolescentes. Por otro lado, el estudio de la historia de la especialización de maestros facilita el entendimiento del porqué de la implementación de ciertas políticas educativas, métodos de enseñanza y medidas disciplinarias para los adolescentes, así como del funcionamiento de las prácticas de dominación de un sector social (adulto) sobre otro con base en regulaciones de la función, los límites y las obligaciones de cada grupo de edad en periodos históricos particulares (Bernardi, 2007).

La información utilizada para este artículo proviene de los fondos Escuela de Altos Estudios y Ezequiel A. Chávez del Archivo Histórico de la UNAM. Es importante mencionar que la recopilación de documentos de la ENAE realizada por Patricia Ducoing en el segundo volumen de La pedagogía en la Universidad de México, 1881-1954 fue de gran ayuda para la localización de fuentes primarias, particularmente de los planes y programas de estudio de dicha institución. Como telón de fondo de la investigación se encuentran las teorías de Bernardo Bernardi (2007) sobre la conformación y funcionamiento de las clases de edad que aparecen en su libro Age class system. Social institutions and polities based on age.

\section{El Estado educador: la formación de docentes}

La tutela de los gobiernos liberales sobre algunos sectores de la población infantil y juvenil a partir de la expansión de la enseñanza básica y de la formación especializada de maestros fue una corriente generalizada en Occidente desde los últimos años del siglo XVIII. Durante los siguientes siglos, como parte de la reorganización política de los Estados nacionales, la educación pública se convirtió en un vehículo para la difusión de las ideas propias del nuevo sistema político. Los proyectos de homogeneidad cultural y lingüística, las nociones de ciudadanía y el nacionalismo resultaron fundamentales para el establecimiento, centralización e institucionalización del poder gubernamental (Anderson, 1993; Gellner, 2006: 63-64; Blom y Stepputat, 2001: 1-38). De acuerdo con la retórica de algunos ideólogos progresistas europeos, el Estado tenía la tarea de administrar la educación con el propósito de impulsar el bien común y el progreso nacional. Era obligación de los gobiernos garantizar la enseñanza de las verdades de hecho, así como alejar a la población del dogmatismo de las órdenes religiosas que habían controlado la educación prácticamente desde el medievo. 
En México, también los gobiernos liberales de mediados del siglo XIX consideraron a los maestros como difusores de los valores de su doctrina política y a las escuelas como espacios óptimos para su transmisión; esto en contraposición con los valores pregonados por la Iglesia católica, que anteriormente estaba a cargo de gran parte de los centros educativos (Agustín, 1982: 55-76). Si bien los primeros esfuerzos por implantar escuelas especializadas en la formación de maestros comenzaron una vez alcanzada la independencia del país, con el establecimiento de las escuelas lancasterianas, fue hasta finales del siglo XIX que los gobiernos nacionales y estatales se involucraron en la preparación de docentes de enseñanza primaria (Tanck, 1992: 49-68). Así se abrieron varias escuelas normales en distintos estados de la República. En la Ciudad de México, pese a las propuestas de Justo Sierra, en 1881, de fundar una escuela normal encargada de la formación de maestros para todos los niveles, esto sólo pudo concretarse unos años después con el establecimiento, en 1885, de la Escuela Normal de Profesores y, en 1888, de la correspondiente para profesoras (Bazant, 1993: 129-146). Dichos planteles se especializaron únicamente en los educadores de enseñanza básica.

El interés gubernamental por la institucionalización de la formación de profesores de enseñanza primaria convergió con las nuevas teorías psicológicas y propuestas pedagógicas estadunidenses sobre la adolescencia a finales del siglo XIX. Algunas de estas ideas fueron aplicadas en los planes de estudios de la Escuela Nacional Preparatoria que albergaba, hasta 1925, año de establecimiento de la escuela secundaria, predominantemente a adolescentes de entre 13 y 19 años de edad, procedentes de los sectores sociales medios y altos de la Ciudad de México y de algunas urbes del país. ${ }^{2}$

Fundada en 1867, la ENP a pesar de ser una institución prácticamente destinada a la educación de los hijos de familias acomodadas (cuya matrícula había variado de entre novecientos a mil alumnos entre su fundación y 1893), recibió durante los primeros años del Porfiriato 68\% del presupuesto gubernamental en educación, lo que generó inconformidad entre algunos políticos y la reducción de dicho monto. (Hale, 2002: 225-226, 246; Guerra, 1993: 426). ${ }^{3}$

Durante el régimen de Díaz, los directivos de la ENP mantuvieron una estrecha comunicación con el Estado para la aprobación, desaprobación y cambios en sus programas de estudio, así como en lo relativo a la contratación de su personal docente, el cual sobresalía más que por su experiencia magistral, por su desarrollo profesional o afiliación política liberal (Lemoine, 1972: 51-52).

\footnotetext{
${ }^{2}$ La mayoría de los jóvenes del país no terminaba su educación primaria y muy pocos podían ingresar a la escuela preparatoria e incluso a las escuelas de artes y oficios. De acuerdo con algunas estimaciones, en 1895 sólo 20\% de la población entre 0 y 15 años asistía a la escuela. (Bazant, 1993:89)

${ }^{3}$ Durante los últimos años del Porfiriato la escuela primaria recibió la mitad del presupuesto designado al ramo educativo.
} 
En las siguientes décadas, algunos funcionarios, como Francisco Vázquez Gómez, Porfirio Parra, Ezequiel A. Chávez, Andrés Osuna, Nemesio García Naranjo y Moisés Sáenz, observaron la importancia de procurar una educación meticulosa, de adecuar los programas de estudio a las capacidades de los estudiantes adolescentes al igual que los métodos pedagógicos y la formación o especialización de profesores para ese nivel de estudios. ${ }^{4}$

\section{La institucionalización y profesionalización de la enseñanza para adolescentes}

En 1881, el joven abogado y funcionario Justo Sierra presentó una propuesta para el establecimiento de una Universidad y una Escuela Normal enfocada en la formación de docentes de todos los niveles frente al Congreso de la Unión (Ducoing, 1990a: 44-60). Aunque el proyecto no prosperó, en 1901, ya como subsecretario de Instrucción, Sierra lo revivió con algunos cambios. En su primer bosquejo, por ejemplo, consideró la formación de maestros para todos los niveles educativos (desde Kindergarten hasta la enseñanza superior); en el último, la Universidad debía incorporar una Escuela Normal encargada únicamente de la preparación de profesores de niveles superiores y profesionales a partir de su formación científica (Ducoing, 1990a: 64-65; Arnaut, 1998: 63). ${ }^{5}$

Fue hasta 1908 cuando la propuesta de Sierra comenzó a tomar forma; en ella, Ezequiel A. Chávez, profesor de la Escuela Nacional Preparatoria y subsecretario de Instrucción, tuvo una importante participación. A él se le encomendó la tarea de elaborar un boceto de la Universidad Nacional de México y de la Escuela Nacional de Altos Estudios. En 1903, 1906 y 1909, Chávez fue comisionado por Sierra para visitar las universidades de Berkeley y Stanford en Estados Unidos y recabar información sobre su estructura y funcionamiento. Dichas observaciones, junto con el modelo de la Escuela de Altos Estudios de Francia, sirvieron como base para el diseño de ambas instituciones universitarias. Esto, aunado al contacto de Chávez con el ambiente académico estadunidense, en el que las políticas educativas sobre la adolescencia se encontraban en boga, despertó su interés por la formación de los individuos durante esa etapa de vida (Chávez, 2002: 234-241).

En 1908, una comisión dictaminadora compuesta por funcionarios y reconocidos pedagogos encargada de evaluar la viabilidad del establecimiento de la ENAE manifestó: ${ }^{6}$

${ }^{4}$ Para más información sobre el papel y las precepciones de cada uno de estos personajes, véase (Meza Huacuja, 2015: 102-125, 148-185)

${ }^{5}$ De hecho, durante el Segundo Congreso de Instrucción Pública (1890), el ministro de Instrucción Pública, Joaquín Baranda, afirmó que el objetivo de las escuelas normales no era formar docentes sino científicos.

${ }^{6}$ Chávez presidió el Consejo Superior de Educación, que tenía como misión el establecimiento de la Escuela de Altos Estudios. Dicha comisión estaba integrada por Porfirio Parra (director de la Escuela Nacional Prepa- 
Que la mencionada institución tendría como objetivo el perfeccionamiento y la especialización de los conocimientos adquiridos en las escuelas preparatorias y profesionales; la formación de un cuerpo de profesores competentes para las escuelas de educación secundaria y profesional; y la organización de investigaciones científicas (Valderrama et al, 1997: 31).

Finalmente, en 1910 Porfirio Díaz expidió la Ley Constitutiva de la Universidad Nacional de México que integró a la Escuela Nacional Preparatoria y a las Escuelas de Jurisprudencia, Medicina, Ingenieros, Bellas Artes y Altos Estudios. Esta última estuvo a cargo de la especialización de los conocimientos adquiridos previamente en las escuelas universitarias, funcionando prácticamente como un centro de estudios de posgrado y de especialización docente en enseñanza secundaria. Durante sus primeros años de existencia la ENAE se dividió en tres secciones: una de humanidades, que incluyó lenguas clásicas, lenguas vivas, literaturas, filología, pedagogía, lógica, psicología, ética, estética, filosofía y doctrinas filosóficas; otra de ciencias exactas, físicas y naturales, y una de ciencias sociales, políticas y jurídicas (Sierra, 1910: 10).

A ella podían inscribirse aquellos que presentaran un certificado de estudios de cualquiera de las escuelas universitarias y que hubieran alcanzado "la más alta calificación" en la materia relacionada con la que fueran a emprender o, en su defecto, quienes obtuvieran buenos resultados ante un jurado nombrado por la ENAE para calificar su aptitud. También podían ingresar estudiantes de las escuelas de los estados cuyos planes y programas de estudio tuvieran equivalencia con los de la Universidad Nacional, al igual que los alumnos de las escuelas particulares y extranjeros quienes debían "someterse [...] a los requisitos que exijan los reglamentos" (Sierra, 1910: 12).

En suma, la ENAE dio prioridad a la instrucción de aquellos individuos que cursaban o habían cursado la Universidad; sin embargo, los normalistas, aunque en menor número en un inicio, también fueron admitidos. Años más tarde, en una carta al rector de la Universidad, Chávez señalaba la edad como una de las razones de dicha particularidad, pues los normalistas prácticamente terminaban sus estudios como maestros de primaria a los diecisiete años, a diferencia de los jóvenes universitarios, quienes en su mayoría concluían la universidad alrededor de los veintiún años:?

[...] las escuelas primarias para maestros reciben, en efecto, alumnos que no cuentan en México con más preparación que la de las escuelas primarias comunes: dicha

ratoria), José A. Aguilera, Alberto Correa, Pablo Macedo, Victoriano Pimentel, Rafael Martínez Freg y Luis Cabrera.

7 Sin embargo, en algunos programas de estudio se permitía la inscripción para obtener la acreditación como profesor universitario a los alumnos que se encontraran cursando sus estudios profesionales. AHUNAM, ENAE, Secretaría Académica, cursos, caja 25, exp. 585, fj. 47 (1922). 
preparación obtenida de un modo normal entre los seis y los doce años de edad, hace que quienes estudian en las escuelas primarias deban principiar allí su carrera más o menos a los trece años, y con esto solo basta para hacerse cargo de los estudios que en las escuelas normales referidas se realicen, deberán ser indispensable de mera iniciación y no de investigación. Por otra parte, dado que la carrera del normalista dura también normalmente cinco años, puede afirmarse que alumnos que hagan en ella sus estudios de un modo regular deberán concluir la referida carrera entre los diecisiete y los dieciocho años, esto es, cuando ni el juicio está suficientemente maduro todavía para poder hacerse cargo de los problemas más graves, ni hay tiempo bastante para afrontarlos, ya que como es bien sabido, seguramente más de la mitad del tiempo que se invierte en los estudios de las escuelas normales no puede gastarse en hacer estudios de filosofía, la ciencia, y el arte de la educación propiamente dichos [... $]^{8}$

No obstante, de manera un tanto contradictoria, algunos egresados de la Escuela Nacional Preparatoria asistieron a dichos cursos desde el establecimiento de la ENAE hasta 1924. A diferencia de los otros dos casos, y de acuerdo con ciertos documentos, por lo menos desde 1916 fueron obligados a tomar cursos "propedéuticos" en una disciplina de su propia elección que los prepararía para su especialización como profesores de preparatoria en dicha materia. Por su parte, algunas autoridades universitarias consideraron que su futuro desempeño como docentes apoyaría a los estudiantes económicamente por un corto periodo, pues se esperaba que prosiguieran con sus estudios profesionales en la disciplina seleccionada (Universidad Nacional de México, 1918).

La irrupción de la Revolución mexicana trajo consigo nuevas concepciones sobre el rumbo de la educación. En 1913, ya como director de la ENAE, Chávez emprendió ciertos cambios en la estructura de dicha institución: por un lado, integró como profesores a algunos miembros del Ateneo de la Juventud, como Alfonso Reyes, Pedro Henríquez Ureña, Antonio Caso y Luis G. Urbina y, por el otro, fortaleció los programas de estudio para la formación de maestros de enseñanza superior (Ducoing, 1990a: 118). Poco a poco, la preparación de docentes se volvió una actividad más compleja, aunque con materias humanísticas fue predominantemente científica y cada vez más institucionalizada. Además de aprobar las asignaturas de especialidad de acuerdo con la disciplina de estudios seleccionada, los alumnos inscritos tenían que tomar un curso de ciencia, arte de la educación y psicología general, además de practicar la docencia en alguna institución educativa (Reidi y Echeveste, 2004: 5).

El establecimiento de la Secretaría de Educación Pública, en 1921, fue la concreción del proyecto de regeneración y justicia social por medio de las políticas de federalización de la educación (centralizadoras) formulado por José Vasconcelos. Dentro de estas propuestas, los maestros fueron concebidos como el engranaje principal que garantizaría la difusión de

${ }^{8}$ AHUNAM, ENAE, Secretaría Académica, cursos, caja 25, exp. 585, fj. 47 (1922). 
conocimientos utilitarios y de la moral social; su desempeño jugaría un papel capital como motor del progreso y la regeneración nacional. ${ }^{9}$ Durante los siguientes años, Vasconcelos emprendió una serie de reformas en las escuelas normales del país, buscando la uniformidad de la preparación de maestros en todo el territorio nacional y la apertura de un mayor número de instituciones de este tipo (Fell, 2009: 116-121).

Al tener como escenario dicho contexto, y tomando en cuenta el afán centralizador y reorganizador del Estado mexicano durante la década de 1920, no resulta sorpresivo el intento por acercar a la Universidad a los intereses de los sectores desfavorecidos, a los que, de acuerdo con Vasconcelos, la máxima casa de estudios había permanecido ajena (Fell, 2009:18). De esa forma podemos entender la reestructuración, en 1924, de la Facultad de Altos Estudios, desde entonces denominada Facultad de Filosofía y Letras; y la expedición del decreto de Emilio Portes Gil, presidente provisional del país, de fundar la Escuela Normal Superior (ENS) dentro de la Universidad a partir de los cursos pedagógicos impartidos en Altos Estudios. ${ }^{10}$ En esta coyuntura tomaron forma las discusiones sobre la importancia del establecimiento de una escuela de transición entre la primaria y la preparatoria, que además debía promover la asistencia de un mayor número de jóvenes de los sectores populares. Por mandato de las autoridades de la SEP y de la mano de Moisés Sáenz, el subsecretario de Educación, el proyecto fue aprobado a finales de 1925.11 Tras dicho decreto las escuelas secundarias fueron oficialmente establecidas en México con un novedoso programa de tres años enfocado en la enseñanza laica y la cultura general, adaptado a las "capacidades" de los adolescentes. El plan de estudios de las preparatorias (ente ellas, la Escuela Nacional Preparatoria) se redujo de cinco a dos años; sus objetivos fueron los mismos que desde su fundación: la preparación de los jóvenes para entrar a la Universidad y recibieron a los adolescentes egresados de las escuelas secundarias.

Como lo demuestran los objetivos de la ENS, la formación de los educadores de adolescentes continuó siendo tarea de la Universidad por lo menos entre 1924 y 1934, pese a la evidente rivalidad entre las autoridades universitarias y la SEP, que seguía enfocando sus esfuerzos en la educación rural y popular. De acuerdo con los planes de estudio de 1924, en los que habían participado Chávez y Vasconcelos, la ENS tenía como objetivo ofrecer certificados de estudio a profesores de materias especiales en escuelas normales, secundarias o preparatorias, además de cursos complementarios y de especialización como directores e inspectores de escuelas primarias y secundarias para los egresados de la Normal de

\footnotetext{
${ }^{9}$ El concepto de moral social se refería a la capacidad del individuo de discernir entre lo bueno y lo malo, a la responsabilidad sobre sus acciones y a luchar por alcanzar la justicia social (Fell, 2009).

${ }^{10}$ AHUNAM, ENAE, Dirección, Acuerdos y Circulares, caja 6, exp. 125 (1924).

${ }^{11}$ Engracia Loyo, "De la desmovilización a la concientización. La escuela secundaria en México, (1925-1940)" en <biblioweb.tic.unam.mx/diccionario/htm/articulos/sec_7.htm> (consultado el 15 abril de 2016).
} 
Maestros. ${ }^{12}$ Dentro de las materias obligatorias que los futuros docentes debían cursar se encontraban: "Ciencia de la educación que comprenda psicología de la adolescencia y la educación relacionada con nuestros problemas económicos y sociales", impartida por Chávez desde 1922; y "Orientación y organización de escuelas normales" por Manuel Barranco y Lauro Aguirre u "Orientación y organización de escuelas secundarias y preparatorias", dictada por el subsecretario de Educación Pública, Moisés Sáenz, así como "Técnicas de enseñanza de la materia objeto de especialización" (Ducoing, 1990a: 182).

Por su parte, los futuros directores e inspectores, además de los cursos mencionados, debían inscribir: "Organización y administración escolar", impartida por Manuel Barranco; "Psicología educativa", por Alfredo E. Uruchurtu; "Estimación crítica de aptitud, aprovechamiento y desarrollo mental”, por Carmen Ramos, Emma Bernal, Ana María Gómez y Montana Hastings; "Historia crítica de la educación en México", por Juana Palacios; "Curso superior de Higiene Escolar", por Rafael Santamarina, y "Curso especial de estimación crítica del desarrollo mental de niños anormales y delincuentes", por David Pablo Boder. ${ }^{13}$

En los años 1926, 1928 y 1930 se realizaron pocas modificaciones a los planes de estudio, los programas fueron prácticamente los mismos que en 1924, aunque con la adición de "Sociología de la educación" como materia optativa. En los cursos para obtener certificaciones como directores e inspectores sí hubo cambios mayores; se insistió en la importancia del dominio de las técnicas pedagógicas con asignaturas como "Principios de educación en escuelas primarias" y "Técnica de enseñanza", y se agregaron materias auxiliares como "Psicología de la adolescencia", "Estadística" y "Sociología de la educación", entre otras.

A los pocos meses de su fundación, en 1921, la rivalidad entre la SEP y la Universidad fue evidente, particularmente respecto al papel de una y otra institución en la toma de decisiones sobre el rumbo de la educación pública en el país. ${ }^{14}$ En el campo de la preparación docente, la pugna se centró en el control de la formación de los adolescentes y se materializó en la separación de la ens de la Facultad de Filosofía y Letras y su anexión a la Secretaría de Educación, en 1934. Dicha escisión coincidió con los discursos de democratización de la educación superior durante el gobierno cardenista y con su interés por formar jóvenes solidarios con los sectores trabajadores y la comunidad en general (Zorrilla, 2004: 5). Pese a

12 "Acuerdo para el señor rector de la Universidad y el C. Secretario de Educación Pública". AHUNAM, ENAE, Dirección, Acuerdos y circulares, caja 6, exp. 124, fos. 5, 929. En una segunda fase de su organización académica participaron Pedro Sánchez, Isaac Ochoterena, Enrique O. Aragón, Alfonso Caso, Carmen Ramos y Balbino Dávalos (Ducoing, 1990a:180)

13 José Vasconcelos, "Proyecto de reorganización de la Facultad de Altos Estudios conforme a las disposiciones dictadas por la Secretaría de Educación Pública el 23 de enero de 1924", (1924).

14 Podría agregarse la desconfianza de algunos funcionarios hacia los jóvenes universitarios capitalinos, quienes además de su aparente falta de apoyo al conflicto armado y a las políticas revolucionarias, parecían renuentes a involucrarse en las políticas educativas y la asistencia social a los sectores menos favorecidos. Para más información véase Garciadiego, 1996. 
dichas resoluciones, los maestros normalistas y profesores universitarios compartieron la tarea de formar a los adolescentes de las escuelas secundarias públicas y privadas (Ducoing, 1990a: 225). Por su parte, los profesores egresados de la Universidad mantuvieron su prerrogativa sobre la educación de estudiantes de la ENP.

\section{El perfil del maestro: ideales y realidades}

Como ya se señaló, desde 1910 hasta 1921 la especialización de profesores de enseñanza secundaria pública en Altos Estudios sirvió como una forma de superación para los egresados de la Escuela Normal de Maestros y como una fuente de empleo temporal y una manera alternativa de desarrollar su profesión a largo plazo para los universitarios. Ahora bien, como parte de los esfuerzos de centralización educativa, la preparación apropiada de los docentes y su homogeneización fueron implementadas por la SEP, en 1923, como requisito para lograr la "revalidación de estudios" de las escuelas particulares..$^{15}$ Así pues, aunque los documentos de registro de alumnos no mencionan la institución de procedencia, salvo que la gran mayoría residían en el Distrito Federal, puedo inferir, apoyada en algunas disposiciones oficiales, que una parte considerable de los inscritos en la ENS eran profesores de escuelas privadas (Torres, 2004). ${ }^{16}$ Cabe destacar que desde 1929 se establecieron cursos de verano enfocados en la preparación de profesores de enseñanza secundaria de los estados.

Los cambios más trascendentes en la formación de los docentes en relación directa con las nociones de adolescencia moderna pueden ubicarse, en 1922, con el establecimiento de la cátedra de "Psicología de la adolescencia". No obstante, fue hasta 1924 cuando se incorporó como materia obligatoria de la especialidad para ejercer como maestros, directores e inspectores de escuelas secundarias. Es muy evidente que dichas modificaciones mantuvieron un vínculo profundo con las propuestas para la instauración de la escuela secundaria nacional. Incluso puede argumentarse que la ENS surgió como espacio de homogeneización y formación en pedagogía especializada, y también como una institución encargada de acercar a sus estudiantes con los nuevos planes de estudio, con las preocupaciones sobre la adolescencia y con los estudiantes, pero, sobre todo, con las políticas educativas centralizadoras, democratizadoras y difusoras de las nuevas concepciones nacionalistas y de justicia social:

15 "Resoluciones aprobadas por el Consejo Universitario el 21 de mayo de 1922", en Boletín de la SEP, 1 de enero de 1923, p. 192.

${ }^{16}$ No pude encontrar registro alguno de escuelas privadas enfocadas en educación superior en 1923. Sin embargo, durante los siguientes años en las Memorias de la SEP se encuentran registrados algunos planteles. En dichos registros es evidente que el número de colegios privados es mayor que el de escuelas públicas, aunque mantuvieron un bajo número de inscripciones. 
La escuela nueva exigía nuevos maestros; profesores que no fueran indiferentes, que convivieran con los alumnos que quitaran a las cátedras el sello de arcaico dogmatismo y se desarrollaran en un ambiente de serenidad socrática; que el maestro en sus explicaciones no fuera más que el guía, y se impulsara y se diera importancia en cambio, al trabajo personal del alumno, al predominio de la actividad; que se enseñara a observar y se le condujera a las fuentes del saber para que la enseñanza muerta del recitador fuera sustituido por la educación viva y racional; que en vez del texto único, del "libro de horas" se le facilitara la lectura de obras científicas diversas, de tratados de historia, etc., y como complemento la redacción de notas por los mismos alumnos en las que sintetizaran la verdad o verdades encontradas y el saber adquirido, único que sobrevive al tiempo y al olvido. ${ }^{17}$

Durante estas fechas hasta 1934 la especialización docente no fue considerada como un requisito obligatorio para impartir clases en las escuelas públicas, pero sí para obtener nombramientos oficiales como directores e inspectores de los planteles. Las autoridades educativas de la SEP alentaron a los egresados de la normal de maestros a tomar cursos en la ENS como una forma de incrementar sus ingresos económicos dentro del sistema de escalafón (SEP, 1926: 3-22, 171-177). De igual forma, los directivos de la Universidad, en un intento por terminar con la inasistencia de los profesores preparatorianos, ofrecieron una mejora salarial a aquellos que perfeccionaran su especialidad y se comprometieran tiempo completo con la institución (SEP, 1925: 226-228). Sin embargo, de acuerdo con algunas declaraciones de los mismos maestros, en 1933 los salarios permanecían bajos y la saturación de las clases con 50 alumnos y hasta 12 grupos por profesor interfería en la calidad de los cursos. ${ }^{18}$ Pervivían, además, prácticas de compadrazgo político y recomendaciones para obtener puestos docentes y directivos. ${ }^{19}$

Según algunos datos proporcionados por la SEP, entre 1925 y 1932 las inscripciones anuales en la ENS doblaron e incluso triplicaron a las de la Facultad de Filosofía y Letras y la Facultad de Graduados (a excepción de 1929 y 1933; Ducoing, 1990a: 177) ${ }^{20}$ Los porcentajes derivados de los registros oficiales hacen presumir que los maestros especializados sobrepasaban los lugares disponibles en las escuelas secundarias públicas y privadas, pese a los

17 "Los maestros de planta", en Revista de Educación, junio de 1933, año 1, núm. 5, p. 162.

18 "Los maestros de planta", Revista de Educación, junio de 1933, año 1, núm. 5, p. 162.

19 "Urge la ley de escalafón", Revista de Educación, julio de 1933, año 1, núm. 6, pp. 201-202.

20 Estos datos pertenecen a 1925: la Facultad de Filosofía y Letras contaba con 317 inscritos, la Escuela de Graduados con 42 y la Normal Superior con 810. También cabe destacar que según algunos documentos, en 1926, los alumnos de la ENS tenían que pagar cuatro pesos por asignatura para presentar cada examen final. "A estudiantes universitarios" (1926), AHUNAM, ENAE, Dirección, Acuerdos y circulares, caja 6, exp. 119, fj. 101. En 1929 es posible encontrar una cuota de inscripción de cinco pesos por "cualquier número admisible de materias" para alumnos regulares y de cinco pesos por asignatura para visitantes. AHUNAM, ENAE, Dirección, Secretaría. Académico, planes y programas de estudio, caja 21, exp. 474, Fj. 3. 
bajos niveles de graduación (véase cuadro 1). ${ }^{21}$ De ser así, ¿cuál era la principal motivación por la que los egresados de la Escuela Normal de Maestros y los universitarios asistían a dicha especialización? ¿Existió un interés genuino por mejorar el sistema educativo o entender la problemática adolescente? ¿O simplemente los maestros guardaron la esperanza de que cursando la especialidad podían asegurar un trabajo estable en las instituciones educativas urbanas de reciente fundación?

En un intento por contestar algunas de estas preguntas he elaborado el siguiente cuadro basándome únicamente en las escuelas secundarias, puesto que la Escuela Nacional Preparatoria contrató, en su mayoría, a profesionistas egresados de las facultades universitarias, sin necesariamente haber tomado cursos de especialización en la ENS:

\section{Cuadro 1}

\section{Cuadro comparativo docentes/alumnos inscritos}

\begin{tabular}{|c|c|c|c|c|c|c|c|}
\hline Año & $\begin{array}{l}\text { Núm. de } \\
\text { alumnos } \\
\text { inscritos a } \\
\text { la ENS[1] }\end{array}$ & $\begin{array}{l}\text { Núm. de } \\
\text { secundarias } \\
\text { públicas en } \\
\text { el D.F. }\end{array}$ & $\begin{array}{l}\text { Núm. de } \\
\text { alumnos } \\
\text { escuelas } \\
\text { públicas en } \\
\text { el D.F. }\end{array}$ & $\begin{array}{l}\text { Núm. de } \\
\text { profesores de } \\
\text { secundarias } \\
\text { públicas en el } \\
\text { D.F. }\end{array}$ & $\begin{array}{c}\text { Núm. de } \\
\text { escuelas y } \\
\text { preparatorias } \\
\text { particulares.* }\end{array}$ & $\begin{array}{l}\text { Núm. de } \\
\text { alumnos de } \\
\text { escuelas } \\
\text { preparatorias } \\
\text { particulares * }\end{array}$ & $\begin{array}{l}\text { Núm.de } \\
\text { profesores de } \\
\text { escuelas } \\
\text { preparatorias }\end{array}$ \\
\hline 1925 & 810 & - & - & - & s.d. & s.d. & s.d. \\
\hline 1926 & 1389 & 4 & 3860 & 357 & - & - & - \\
\hline 1927 & 811 & 5 & 4729 & s.d. & $11^{*}$ & $1,201^{*}$ & $258^{*}$ \\
\hline 1928 & 900 & $6+1$ noct & 5889 & 438 & $15^{*}$ & 1,736 & $353^{*}$ \\
\hline 1929 & 574 & 6 & 5543 & s.d. & $\begin{array}{c}19^{\star} \\
\text { (14 en D.F.) }\end{array}$ & 2,128 & $269^{*}$ \\
\hline 1930 & $648^{\star \star}$ & 7 & 6005 & s.d. & $\begin{array}{c}20 \\
(15 \text { en D.F. })\end{array}$ & 2,223 & s.d. \\
\hline 1931 & $1031^{\star \star}$ & 8 & 7009 & s.d. & 32 & 2,325 & s.d. \\
\hline 1932 & $1024^{\star \star}$ & 9 & 6885 & $527^{\star}$ & s.d. & 2,978 & s.d. \\
\hline 1933 & $796^{\star \star}$ & 10 & 6193 & $561^{*}$ & 33 & $3661^{*}$ & s.d. \\
\hline
\end{tabular}

Fuente: (Ducoing, 1990a: 177, 203), (SEP, 1925; SEP, 1926; SEP, 1928; SEP, 1929; SEP, 1934). * Corresponden a datos a nivel nacional. No se encontró información específica sobre el Distrito Federal. ** Fue imposible separar el número de maestras que asistían a cursos como maestras de kindergarten y de los directores de escuelas primarias. 1) Vale la pena destacar que en esa cantidad se incluyen las profesoras que asistieron también a los cursos de especialidad en kindergarten, profesores que deseaban ejercer como directores de escuela primaria y o como docentes para las escuelas normales (Ducoing, 1990, 1: 177, 203).

${ }^{21}$ En 1923, por ejemplo, estaban inscritos en la ya para entonces Facultad de Altos Estudios, 859 alumnos, con una asistencia media de 920.5 (de acuerdo con las autoridades de la institución no todos los asistentes "formalizaban su inscripción"); 89 alumnos examinados y 88 aprobados. AHUNAM, ENAE, Estadística, caja 16, exp. 335 fj. 6. Con el establecimiento de la ENS, estas cifras se transformarían. En 1925, los alumnos inscritos fueron 1169 (de los cuales 953 eran mujeres), mientras que, como oyentes, asistían 119, el número de examinados ascendía a 812; aprobados a 785, y los titulados a 3 (un varón y dos mujeres). AHUNAM, ENAE, Estadística, caja 16, exp. 337, fj. 62. 
Una de las primeras peculiaridades que se pueden observar en la tabla es que el número de profesores que ingresaron a los cursos especializados en formación de adolescentes era superior a la cantidad de alumnos y de escuelas secundarias establecidas en México. Incluso, si se toma en cuenta la existencia de aproximadamente treinta materias en total en el mencionado nivel educativo, el número de maestros requeridos era mucho menor al de alumnos inscritos a en la ENS.22 No obstante, la falta de constancia de los futuros especialistas era visible en las bitácoras de la Escuela, en las que se registraba la asistencia y calificaciones de los alumnos. Así pues, puedo inferir que un número limitado de alumnos eran los que lograban ser certificados, lo cual puede explicar algunos aspectos de la saturación de educandos por cada maestro de dicho nivel.

Respecto a otros datos presentados es importante resaltar que en 1927 hubo una reducción en el presupuesto para las escuelas secundarias públicas, por lo que sus directivos tomaron la decisión de cesar a algunos profesores y limitar el número de inscripciones por plantel. ${ }^{23}$

De acuerdo con El esfuerzo educativo, en 1928, tras dos años de comenzar a funcionar las escuelas secundarias públicas, entre los docentes había: 128 normalistas, 28 médicos, 39 ingenieros, 20 abogados, 47 con otros títulos y 112 sin título profesional (SEP, 1928: 388). Una distribución semejante podía encontrarse en las escuelas particulares; según la memoria de 1929 estaban registrados: 15 abogados, 11 médicos, 12 ingenieros, 4 arquitectos, 88 normalistas, un escultor, 22 bachilleres, 80 con otros títulos y 36 sin título alguno (SEP, 1929: 209).

Por lo tanto, al analizar el cuadro y la información proporcionada puede asumirse que, a pesar de los esfuerzos por difundir la especialidad y mejorar la educación secundaria, sólo un pequeño sector de los maestros que se inscribían en la ENS se incorporó a las secundarias y preparatorias de la capital, y además que no todos los docentes que impartían clases en ambas instituciones contaban con la capacitación adecuada.

La respuesta a la pregunta sobre las motivaciones de los maestros de escuelas secundarias públicas para inscribirse en los cursos no es del todo concluyente. Pocos son los testimonios que hacen alguna referencia a su interés por el "fenómeno" de la adolescencia, otros pocos - aunque quizás bastante representativos por su participación activa en la organización política magisterial — manifestaban su preocupación por el mejoramiento de las técnicas educativas, por la formación de los adolescentes y por el progreso del país; mientras que

22 Por ejemplo, en 1927 en el primer año de secundaria se impartieron: aritmética, lengua castellana, botánica, geografía, física, inglés o francés, dibujo constructivo, modelado, música y orfeón, oficio, y juegos y deportes. En el segundo año: álgebra y geometría plana, física, zoología, geografía universal y de México, lengua castellana, inglés o francés, dibujo de imitación, música y orfeón, juegos y deportes. En el tercero: geometría en el espacio y trigonometría, química, anatomía (fisiología e higiene), historia general, historia de México, civismo, literatura castellana, música y orfeón, juegos y deportes (SEP, 1928: 394-396).

23 "Acuerdo" en Boletín de la Secretaría de Educación Pública, 1 de marzo de 1927, p. 369. 
otros parecen haber estado convencidos de la utilidad de la especialización tras haber terminado los cursos. ${ }^{24}$

\section{Las asignaturas: el caso de la clase de psicología de la adolescencia}

El plan de estudios para los futuros profesores de secundarias y preparatorias en la Escuela de Altos Estudios fue transformándose con el tiempo. Inicialmente, el peso en la formación de los denominados profesores universitarios (luego profesores de escuelas secundarias) se encontraba en las materias de especialización que eran acompañadas con un curso de dos años de ciencia y arte de la educación, psicología y metodología general. Posteriormente, esta tendencia se invirtió, y las disciplinas pedagógicas abarcaron la mayor parte del currículum, mientras que los cursos prácticos y de especialización estaban constituidos por una o dos materias (Ducoing, 1990b)

Las únicas disciplinas obligatorias que permanecieron en los planes de estudio desde la fundación de la ENS, desde 1924 hasta 1934, fueron "Psicología educativa" y "Psicología de la adolescencia". Subsecuentemente, como ya se mencionó, se añadieron pedagogía, sociología, estadística, mediciones mentales, organización de escuelas secundarias, higiene escolar, por mencionar algunas.

Para la "nueva" institución fue una prioridad enseñar a los maestros cómo transmitir el conocimiento y orientar adecuadamente a los adolescentes, y no la erudición docente sobre un área de estudios específica. Dichos planteamientos iban acordes con las ya tradicionales discusiones sobre si la función de la escuela era la instrucción o la educación, en las que la segunda consideración dominaba (en este sentido, el cambio de denominación de la Secretaría de Instrucción Pública por el de Secretaría de Educación Pública). Es decir, la educación era concebida como el medio ideal para la formación de ciudadanos y no únicamente para la transmisión de conocimientos; esta orientación se convertiría en una de las características medulares de la educación posrevolucionaria (Muñoz, 2005: 44-63).

Por razones de extensión y de pertinencia respecto al tema de este artículo me enfocaré únicamente en el curso de psicología de la adolescencia impartido por Chávez (en ocasiones teniendo como ayudante al psicólogo letón David Pablo Boder (Valderrama, et al., 1997: 49). La importancia de esta cátedra radica en el interés que su titular puso sobre los adolescentes y en haber constituido el primer esfuerzo gubernamental por intervenir en este grupo de edad

${ }^{24}$ AHUNAM, EACH, Producción intelectual y docencia, Psicología, Documentos de su actividad docente, caja 78, exp. 239, fjs. 1-231 (1932-1934) y Revista de Educación, los números consultados comprendieron febrero de 1933 a marzo de 1934. 
partiendo de las propuestas y recomendaciones internacionales sobre su formación. También fue relevante por el papel que Chávez tuvo, por medio de la impartición de dicha materia, en la especialización de varias generaciones de individuos en el tratamiento de los adolescentes, así como por la difusión de tipologías sobre su comportamiento correcto-incorrecto y de conceptos dicotómicos, como normalidad-anormalidad, moralidad-inmoralidad y saludenfermedad en el desarrollo de los jóvenes expuestos en algunas sesiones de su curso y en su libro Ensayo de psicología de la adolescencia (1928). Cabe destacar que dicha obra fue considerada el libro de texto de la asignatura y de acuerdo con una revisión de ambos - curso y publicación - seguían la misma estructura. ${ }^{25}$

El curso de psicología de la adolescencia, por lo menos en 1931, constaba de cuatro partes. En la primera, intitulada "Adolescencia y la vida", Chávez abordaba algunas definiciones científicas de la adolescencia y exponía desde el punto de vista fisiológico y psicológico los cambios que ocurrían durante dicha etapa, que por cuestiones prácticas y de estandarización, se concibió entre los doce años y veintiún años (Chávez, 1928: 22-23), ${ }^{26} \mathrm{La}$ segunda, "Inferencias y corolarios", estaba enfocada más bien en los métodos para estudiar a los adolescentes y en breves observaciones del especialista sobre el caso particular de los "jóvenes" mexicanos. La tercera llevó por título "Los fenómenos cardinales de la vida mental" y su contenido era más extenso; se enfocaba particularmente en la descripción y estudio de las emociones y conductas que tenían lugar durante dicho periodo de vida como el impulso sexual, el instituto autoasertivo, gregario y maternal, así como la imaginación y el desarrollo mental. Por último, la cuarta, "La estructura superior de la vida psíquica", era una compilación de inquietudes referentes al ámbito de la moral que se relacionaban con el cultivo de la religión, el deber y el civismo como elementos fundamentales para el desarrollo óptimo de los adolescentes. Entre ellas, pueden resaltarse las preocupaciones de Chávez por la supuesta pérdida de la feminidad y masculinidad en varones y señoritas, la falta de respeto de los adolescentes respecto de las mujeres, la tendencia a adoptar vicios, así como la perpetración de robos y otros crímenes, y, por lo tanto, la desobediencia de las leyes y reglamentos (Chávez, 1928: 406-409):

Un grave peligro hay que apuntar aquí: peligro que acrecienta el carácter mismo esencial de la adolescencia: si la figura ideal [Chávez se refiere a un personaje o individuo que el adolescente admira] se empaña, si en parte se borra, la naturaleza excesiva de los adolescentes, su frecuente inestabilidad emocional, sus bruscas alternativas

${ }^{25}$ AHUNAM, EACH, Producción Intelectual y docencia, Psicología, Documentos de su actividad docente, caja 78, exp. 238, doc. 6, fjs. 13-15.

${ }^{26}$ De hecho, la definición de Chávez fue considerar la versatilidad de su inicio, en algunos individuos comenzaba con los cambios físicos de la pubertad a los nueve años y medio, diez, once, doce, trece o catorce y su conclusión a los dieciocho, aunque en casos extraordinarios hasta los veinticinco años (Chávez, 1928: 22). 
de irresolución, y de resoluciones no pocas veces irreparables, de las que es tipo Hamlet, fácilmente los llevan al desvío, a la indiferencia; más lejos quizás: si han reverenciado, acaso escarnezcan y se mofen; tal vez cometan crímenes; y en iracundo desconsuelo de lo que crean es su decepción, se entreguen luego, con sombría rabia, o con altanero desdén, a la vida disoluta e impura, al desenfreno.(Chávez, 1928: 341-342).

De acuerdo con Chávez, durante el año escolar de 1931 se pondría mayor atención en los fenómenos de la vida mental, además se solicitaría a los estudiantes que contribuyeran con observaciones sobre su propia adolescencia, la de sus alumnos o conocidos. Según sus apuntes personales requeriría:

Para comenzar esta clase, puesto que [...] Psicología de la Adolescencia y que no se puede hacer ningún estudio de Psicología si no es por medio de la introspección, invita a los alumnos para que se introspeccionen [sic] y describan a sí mismos el hecho cardinal de su vida psíquica, a saber que hay en cada uno una energía recóndita, personal, constante y simplificadora de su [ilegible] que todo lo vivido reconoce que en él se manifiesta con mejor o menor intensidad sea que este dormido o despierto durante toda su vida $[\mathrm{sic}]{ }^{27}$

Las preguntas de algunos exámenes del curso nos acercan al proceso de formación de los profesores de enseñanza secundaria, así como a las preocupaciones de ciertos individuos e instituciones en relación con el efecto del medio social en la constitución de los adolescentes. Por ejemplo, en la evaluación final de 1931, Chávez realizaba los siguientes cuestionamientos:

[...] ¿ha observado usted manifestaciones del instinto autoasertivo en usted mismo, o en adolescentes, o en niños?, ¿qué observaciones ha hecho usted acerca de la influencia que tengan libros, canciones, ilustraciones, exhibiciones cinematográficas, bailes sobre el desarrollo de los instintos combativo, autoasertivo, sexual, socializante, adquisitivo de los adolescentes y los niños y que juicio se forma usted a su respecto? O ¿ha tenido usted ocasión de observar alguna deformación educativa de funestos efectos sociales, que resulte de determinadas enseñanzas en las escuelas o de sugestiones del ambiente [sic]? ¿Cuál o cuáles son las deformaciones de esa naturaleza, que usted ha observado? (Chávez, 2002:284)

Estas preguntas nos permiten apreciar que a Chávez no le interesaba que sus alumnos memorizaran conceptos y teorías psicológicas; más bien, como seguidor de las propuestas pedagógicas de algunos especialistas, como las de los estadunidenses William McDougall,

${ }^{27}$ AHUnAm, EACH, Producción Intelectual y docencia, Psicología, Documentos de su actividad docente, caja 78, exp. 238, doc. 7, fj. 16 
G. Stanley Hall, y John Dewey, y los franceses Jean Piaget y Pierre Janet, entre otros, su propósito era que aprendieran su aplicación para interpretar e incidir en la educación de los jóvenes (Chávez, 1928: 410). Las respuestas de estos exámenes y tesis, muchas de ellas testimonios personales, sirvieron al funcionario para construir tipologías y realizar algunas aseveraciones acerca de la adolescencia y los adolescentes en México. Aunque representan una interesante recopilación sobre la vida de los "adolescentes" durante las décadas de los veinte y los treinta, estas percepciones en muchos casos estaban influidas por conceptos y valoraciones (y prejuicios) que Chávez había transmitido a sus alumnos en su curso.

Pero, ¿cuál era el concepto de adolescencia difundido por Chávez? ¿Por qué la educación de los adolescentes se había convertido en un tema de interés para ciertos sectores de educadores y funcionarios? La respuesta del alumno Miguel A. García nos ofrece una aproximación que conjuga algunas percepciones bastante difundidas entre los especialistas desde prácticamente principios del siglo xx:

El cambio que se opera en el joven que terminando los periodos anteriores de la vida psíquica es tan grande, que para buen número de psicólogos que tratan estos asuntos constituye algo así como un nuevo nacimiento y ven un abismo entre los anteriores periodos psíquicos y el de la adolescencia. Sin embargo, otros muchos autores no ven tal diferencia, sino que la consideran más bien como la consecuencia natural y armónica del desarrollo mental y físico de los anteriores periodos. Sea lo que fuere, el hecho concreto es que en el adolescente aparece un cambio psíquico muy profundo: primero son los grandes cambios fisiológicos que en él se manifiestan: el cuerpo desarrolla de un modo extraordinario y casi repentino, se completa el desarrollo de los órganos característicos de su sexo, los rasgos físicos se fijan definitivamente, la voz se vuelve grave, el rostro viril, etc. Psíquicamente el cambio no es menos profundo [respecto] al niño. ${ }^{28}$

La adolescencia, de acuerdo con Chávez, era la primera de dos partes que componían a la juventud. Se caracterizaba por una serie de transformaciones físicas y psicológicas; era un periodo de introspección y de gran sensibilidad acerca del mundo exterior. Se representaba como una edad crítica y delicada en todos los sentidos. Los esfuerzos físicos o mal ejecutados, se pensaba, provocaban distrofia muscular y el mal funcionamiento de los órganos internos. El medio social, los espectáculos, los conflictos bélicos, las querellas políticas y la relajación de la moralidad podían tener efectos negativos en su comportamiento y convertirse en un problema irremediable durante la adultez (Chávez, 1928: 12, 340).

${ }^{28}$ AHUNAM, EACH, Producción Intelectual y docencia, Psicología, Documentos de su actividad docente, caja 78, exp. 238, doc. 4, fj. 58. 
Una medida muy extendida entre al gunos médicos, psicólogos, educadores y autoridades religiosas mexicanas y extranjeras para el control de los adolescentes fue la vigilancia y dirección adulta. Ésta debía recaer, preferentemente, en individuos especializados en dicha etapa de la vida. Los docentes de enseñanza secundaria formaron parte de dicho sector, cuyo trabajo con las jóvenes generaciones debía repercutir en el progreso de las naciones y de la humanidad:

Este libro intenta traer su contribución al conocimiento de los adolescentes para que, unidos al cabo con sus educadores, se encaminen a la realización del fin último de todo esfuerzo educativo orientado, y aporten, en consecuencia, su concurso, para organizar la armonía que urge imponer a las antagónicas fuerzas entre las cuales se divide el mundo; no sólo a fin de evitar que éste llegue a despedazarse, como tantos ilustres pensadores hoy lo temen, sino con el de rehacer la cultura, restituyendo así la posibilidad de que todos los pueblos, y especialmente México, progresen (Chávez, 1928: 14).

Además, el interés y las preocupaciones por la adolescencia convergieron en México con la difusión de las ideas eugénicas. El mejoramiento de la raza, las campañas contra las enfermedades venéreas y el alcoholismo, así como los esfuerzos por refrendar la orientación sexual de los individuos, formaron parte de una serie de acciones con las que se buscaba incidir en el denominado "mejoramiento" de la sociedad mexicana (Suárez, 2005). En este tenor, a través del curso de "Psicología de la adolescencia", los maestros fueron entrenados para canalizar a los estudiantes con especialistas ante cualquier sospecha de anormalidad mental, "desviación sexual", problema de aprendizaje o enfermedad física (Chávez, 1928: 419). El curso ofrecía parámetros para la observación y detección de irregularidades y medidas profilácticas, y también para encauzar correctamente la energía y el tiempo libre de los adolescentes.

Chávez consideraba a su curso y a "Principios fundamentales de organización y funcionamiento de las escuelas secundarias" como los esfuerzos de mayor trascendencia en México respecto a la formación y estudio de la adolescencia (Chávez, 1928: 131-132). Para algunos profesores, como la señorita Paula Gómez, la utilidad de los cursos de Chávez debía complementarse con la convivencia diaria con los alumnos y profesores, pues el mayor aprendizaje se generaba en las conversaciones con los docentes de los planteles y no en los libros de texto (Alonso, 1933: 137). Otros, como la profesora Dolores Echeverría, directora de una secundaria privada femenina, veían los cursos de Chávez como un recurso fundamental, por sí mismo, para el mejoramiento de la calidad educativa en el país: 
He adquirido la convicción de que, para los maestros de escuelas secundarias y preparatorias, no sólo es importante el estudio de la Psicología de la adolescencia, sino que es un deber, ya que todo Maestro debe conocer a los alumnos que pretende instruir y educar.

No es necesario en un país, que se multipliquen las escuelas y los maestros, si no llenan su cometido; lo que es una necesidad imperiosa es mejorar la calidad de ambos.

Si la educación se realiza de un modo atinado, pueden prevenirse grandes males, pero si por el contrario, se imparte de un modo vicioso, nada puede perjudicar tanto a los individuos como a los pueblos con una viciosa educación. Un país no está en vías de progreso por el sólo hecho de multiplicar sus escuelas, si estas son instrumentos de desorganización, ese país caminará a la ruina. ${ }^{29}$

En sus reflexiones, Echeverría no sólo resumía ciertas directrices planteadas por las corrientes educativas modernas, como la importancia de conocer el perfil psicológico del alumno fuera niño o adolescente, sino que también criticaba los esfuerzos gubernamentales que pretendían acabar con el analfabetismo abriendo un gran número de escuelas rurales y primarias a lo largo y ancho del territorio nacional.

Como consecuencia de los roces entre la Secretaría de Educación y la Universidad, que era acusada de mantenerse ajena a la resolución de los problemas nacionales, la Escuela Normal Superior fue incorporada a la primera en 1934, enfocándose en ofrecer cursos breves y conferencias para formar profesores de enseñanza secundaria únicamente durante el verano (Castañeda, 2006: 118). En su lugar, la Universidad creó el departamento de ciencias de la educación y, posteriormente, en 1938, la maestría en ciencias psicológicas. En esta última, la clase de Psicología de la Adolescencia permaneció a cargo de Chávez por lo menos hasta 1939 (Valderrama et al., 1997: 25, 32-34). ${ }^{30}$

Por su parte, en 1936, las autoridades de la SEP, en pleno sexenio cardenista, transformaron la ens en el Instituto de Preparación para Maestros de Enseñanza Secundaria en el Distrito Federal, al mismo tiempo que se daba un fuerte impulso a la construcción de más escuelas secundarias (SEP, 1937: 209). ${ }^{31}$ Este instituto sobrevivió, hasta 1940, cuando fue sustituido por el Instituto Nacional de Magisterio de Segunda Enseñanza (Sandoval, 2000: 73). Dos años más tarde, por breve tiempo, se transformó en el Centro de Perfeccionamiento para

${ }^{29}$ AHUNAM, EACH, Producción Intelectual y docencia, Psicología, Documentos de su actividad docente, caja 78, exp. 239, doc. 12, fjs. 228-231, (1934).

${ }^{30}$ Otros profesores que impartieron dicha cátedra dentro de la carrera y luego Licenciatura en Psicología fueron Eduardo Nicol, en 1943; Guillermo Corona H., Agustín Lemus Talavera y Oswaldo Robles en 1962. (Reidi y Echeveste, 2004: 31-34)

${ }^{31}$ En 1936 ya se encontraban establecidas en el territorio nacional 27 escuelas secundarias diurnas y seis para hijos de trabajadores (SEP, 1938: 19). 
Profesores de Enseñanza Secundaria y unos meses después retomó su antiguo nombre de Escuela Normal Superior de México.

\section{Conclusiones}

De acuerdo con las propuestas estructuralistas del antropólogo italiano Bernardo Bernardi, la organización de las clases de edad (en Occidente, por ejemplo. infancia, adolescencia, juventud, edad adulta y vejez) y el papel de cada una, son construcciones vinculadas al contexto cultural y al grupo social en el que se desarrollan (Bernardi, 2007: 7). Aunque Bernardi enfoca su estudio al sistema de grupos de edad en algunas tribus africanas, sus observaciones han sido aplicadas por algunos historiadores, sociólogos y antropólogos en la explicación de las relaciones intergeneracionales de ciertas comunidades en Europa y América, algunas obras son las pertenencientes a Tim Partin (2003, en Meza, 2016) y Laszlo Kurti (2002, Meza, 2016) entre otras. En el caso del presente trabajo, las propuestas de Bernardi han contribuido a reafirmar que la noción moderna de adolescencia surgió en un espacio geográfico particular, como son las zonas urbanas de Occidente, y a que su conceptualización nació de postulados científicos difundidos en Estados Unidos a mediados del siglo XIX.

Por otra parte, la explicación del sistema de clase de edad planteado por Bernardi permite formular, a grandes rasgos, que el imperante en nuestra sociedad urbana está basado en la preponderancia de los sectores adultos sobre individuos de otras edades, particularmente respecto a los de menor edad. Y que la construcción de la noción moderna de adolescencia en Occidente se inscribe dentro de este juego de relaciones, donde el adolescente fue y es representado como un ser inacabado, cuya inestabilidad emocional y susceptibilidad física lo subordina necesariamente a la autoridad adulta.

El presente artículo parte de la idea, abordada en otra de mis investigaciones, que apunta que la educación secundaria en México fue el medio, por excelencia, de difusión la conceptualización de la adolescencia y de los ideales sobre los adolescentes procedentes de los Estados Unidos y Europa adaptados a las necesidades y realidad mexicana (Meza Huacuja, 2015). Mi tema central en este trabajo se enfocó en demostrar cómo los maestros especializados en las escuelas secundarias fungieron como intermediarios entre las expectativas y representaciones que los constructores de las nociones institucionalizadas de adolescencia intentaron difundir entre la sociedad urbana mexicana. Por estas razones, uno de los primeros pasos fue articular dentro de un contexto internacional y nacional el desarrollo de dicha noción, enfocándome en la incorporación, en el ámbito educativo, de la noción cientificista de adolescencia por medio de la construcción de instituciones educativas como la ENAE y ENS, y la formación docente dentro de dichos espacios. 
La presente investigación se encuentra circunscrita a un momento en la historia de la educación en la que la preparación de maestros constituyó una de las acciones gubernamentales en pos de la centralización del poder político durante los regímenes posrevolucionarios. Mediante ella se procuró alcanzar cierto grado de homogeneización cultural e ideológica entre el sector estudiantil (que contribuiría a la permanencia de proyectos nacionales) con base en la formación de docentes especializados en el trato y tratamiento de dicho sector juvenil. De igual forma, en la reafirmación del Estado y los maestros como figuras hegemónicas frente a sujetos en formación" (en desarrollo cuya etapa final era la edad adulta), cuyas actividades necesariamente debían estar bajo tutela de ciertos grupos de edad con algún grado de especialización en su tratamiento.

Así, en el escenario referido los maestros fungieron como sujetos clave en la difusión de las nociones de justicia social, nacionalismo, progreso e incluso identidades de género; se esperaba que como individuos capacitados científicamente y con las modernas tendencias educativas desempeñaran un papel fundamental como mediadores entre el Estado y los educandos, con lo que, de acuerdo con algunos testimonios de la época, se desplazaría a los padres de familia y a la Iglesia católica del papel central que habían tenido en la formación de las jóvenes generaciones (SEP, 1928: XXXIX-LXVIII).

A su vez, cabría agregar, se pretendía que los estudiantes canalizarían las prácticas higiénicas y los conocimientos aprendidos en la escuela a sus hogares; además, indirectamente se esperaba que los adultos coparticiparan en las actividades escolares y extracurriculares de sus hijos como festivales, concursos de canto, campañas antialcohólicas, entre otras, todas ellas con tintes profundamente nacionalistas (SEP, 1928: 407).

La educación de los adolescentes también fue objeto de fricciones entre el gobierno federal y la Universidad. Si bien en un inicio las SEP delegó en la Universidad la educación de los sectores privilegiados, progresivamente fue reclamando lo que consideró su atribución. Esta situación se hizo evidente con la invitación de maestros normalistas y el aumento de la matrícula de este sector en los cursos de especialización docente como profesores de enseñanza secundaria. Quizá dicha querella llegó a su punto culminante con el establecimiento de la escuela secundaria, a finales de 1925, y del Departamento de Enseñanza Secundaria dentro de la SEP, en 1926 y, paulatinamente, con los constantes conflictos y discrepancias de opinión sobre el objetivo de la ENS, que terminarían con su separación de la Universidad Autónoma de México, en 1934. 


\section{Fuentes}

Archivos

Archivo Histórico de la unam, Ciudad de México, México, Fondo: Escuela Nacional de Altos Estudios (AHUNAM, ENAE).

Archivo Histórico de la unam, Ciudad de México, México, Fondo: Ezequiel A. Chávez (AHunAM, EACH).

Bibliografía

(1923), "Resoluciones aprobadas por el Consejo Universitario el 21 de mayo de 1922", Boletín de la Secretaría de Educación Pública, enero, pp. 192.

(1927), "Acuerdo", Boletín de la Secretaría de Educación Pública, marzo, pp. 368-369.

(1933), "Los maestros de planta" Revista de Educación, año 1, núm. 5, junio, pp. 161-162, 193.

(1933), "Urge la ley de escalafón", Revista de Educación, año 1, núm. 6, julio, pp. 201-202, 232.

Alonso Gómez, Paula (1933), "Los maestros y la psicología de los adolescentes", Revista de Educación, año 1, núm. 4, mayo, pp. 137-139.

Anderson, Benedict (1993), Comunidades imaginadas. Reflexiones sobre el origen y la difusión del nacionalismo, Fondo de Cultura Económica, México.

Arnaut, Alberto (1998), La federalización educativa en México: historia del debate sobre la centralización y la descentralización educativa, 1889-1994, El Colegio de México-Centro de Investigación y Docencia Económicas, México.

Bazant, Mílada (1993), Historia de la educación durante el Porfiriato, El Colegio de México, México.

Bernardi, Bernardo (2007), Age class system. Social institutions and polities based on age, Cambridge University Press, Cambridge.

Blom Hansen, Thomas y Finn Stepputat (2001), States of imagination: Ethnographic explorations of the postcolonial state, Duke University Press, Durham.

Cano, Gabriela (1996), "De la Escuela Nacional de Altos Estudios a la Facultad de Filosofía y Letras, 1910-1929. Un proceso de feminización", tesis doctoral, Universidad Nacional Autónoma de México, México.

Castañeda Rincón, Javier (2006), La enseñanza de la Geografía en México. Una visión histórica: 1821-2005, Universidad Autónoma Chapingo, México.

Chávez, Ezequiel (1928), Ensayo de psicología de la adolescencia, Cultura, México.

Chávez, Ezequiel (2002), Obra filosófica y autobiográfica, t. 2, El Colegio Nacional, México.

Ducoing, Patricia (1990a), La pedagogía en la Universidad de México, 1881-1954, tomo 1, Universidad Nacional Autónoma de México, México.

Ducoing, Patricia (1990b), La pedagogía en la Universidad de México, 1881-1954, tomo 2, Universidad Nacional Autónoma de México, México.

Escolano, Agustín (1982), "Las escuelas normales, siglo y medio de perspectiva histórica", Revista de Educación, núm. 269, pp. 55-76.

Fell, Claude (2009), José Vasconcelos. Los años del águila: 1920-1925, Universidad Nacional Autónoma de México, México.

Garciadiego, Javier (1996), Rudos contra científicos. La Universidad Nacional durante la Revolución mexicana, El Colegio de México-Universidad Nacional Autónoma de México, México.

Gellner, Ernest (2006), Nation and nationalism, Cornell University Press, Ithaca. 
Guerra, François-Xavier (1993), México: del antiguo régimen a la Revolución, vol. 1, Fondo de Cultura Económica, México.

Hale, Charles A. (2002), La transformación del liberalismo en México a fines del siglo xIx, Fondo de Cultura Económica, México.

Kurti, Laszlo (2002), Youth and the state in Hungary: capitalism, communism and class, Pluto Press, Chicago.

Lemoine, Ernesto (1972), La Escuela Nacional Preparatoria en el periodo de Gabino Barreda, Universidad Nacional Autónoma de México, México.

Loyo, Engracia (1999), Gobiernos revolucionarios y educación popular en México 1911-1928, El Colegio de México, México

Meza, Ivonne (2015), "La edad difícil. Los adolescentes modernos en la ciudad de México: 1876-1934", tesis de doctorado, El Colegio de México, México.

Muñoz, Alicia (2005), "Ideales y aplicaciones de la enseñanza moderna en México durante el Porfiriato", Revista de la Universidad Autónoma de Yucatán, vol. 20, núm. 233, abril-junio, pp. 44-63.

Partin, Tim. (2003), Old Age in the Roman World: A Cultural and Social History, Universidad Johns Hopkins. Baltimore.

Reidi, Lucy y María Echeveste (2004), La Facultad de Psicología de la Universidad Nacional Autónoma de México. Treinta años a la vanguardia, Universidad Nacional Autónoma de México, México.

Sandoval, Etelvina (2000), La trama de la escuela secundaria: institución, relación y saberes, Universidad Pedagógica Nacional, México.

Secretaría de Educación Pública (1928), El esfuerzo educativo, Secretaría de Educación Pública, México.

Secretaría de Educación Pública (1937), Memoria de la Secretaría de Educación Pública de septiembre de 1936 a agosto de 1937, Secretaría de Educación Pública, México.

Secretaría de Educación Pública (1938), Memoria de la Secretaría de Educación Pública de septiembre de 1937 a agosto de 1938, Secretaría de Educación Pública, México.

Secretaría de Educación Pública (1925), Memoria que indica el estado que guarda el ramo de la educación pública (1925), Secretaría de Educación Pública, México.

Secretaría de Educación Pública (1926), Memoria que indica el estado que guarda el ramo de la educación pública (1926), Secretaría de Educación Pública, México.

Secretaría de Educación Pública (1929), Memoria que indica el estado que guarda el ramo de la educación pública (1929), Secretaría de Educación Pública, México.

Sierra, Justo (1910), "Ley Constitutiva de la Escuela Nacional de Altos Estudios", en Justo Sierra, La Universidad Nacional de México, Viuda de F. Díaz de León, México.

Suárez, Laura (2005), Eugenesia y racismo en México, Universidad Nacional Autónoma de México, México.

Tanck, Dorothy (1992), "Las escuelas Lancasterianas en la ciudad de México: 1822-1842" en Josefina Vázquez, La educación en la historia de México, El Colegio de México, México, pp. 49-68.

Torres, Valentina (2004), La educación privada en México: 1903-1976, El Colegio de México-Universidad Iberoamericana, México.

Universidad Nacional de México (1918), Disposiciones relativas a la Facultad de Altos Estudios, Imprenta Francesa, México.

Valderrama, Pablo et al. (1997), "De la fundación de la Universidad a la creación de la Maestría en Psicología: 1910-1938", 100 años de la Psicología en México, 1896-1996, Universidad Nacional Autónoma de México, México, pp. 29-58.

Zorrilla, Margarita (2004), "La educación secundaria en México al filo de su reforma", Revista Electrónica Iberoamericana sobre Calidad, Eficacia y Cambio en Educación, vol. 2, núm. 1, documento (pdf), disponible en: <www.ice.deusto.es/RINACE/reice/vol2nl/Zorrilla.pdf>, (fecha de consulta: 3/05/2016). 
Loyo, Engracia (s.f.), "De la desmovilización a la concientización. La escuela secundaria en México, (1925-1940)", documento (html) disponible en: <biblioweb.tic.unam.mx/diccionario/htm/articulos/ sec_7.htm> (fecha de consulta: 15/04/2016).

Ivonne Meza Huacuja. sNI Candidato (2017-2019). Es Doctora en Historia por El Colegio de México. Actualmente realiza una estancia posdoctoral en el Instituto de Investigaciones sobre la Universidad y la Educación (IISUE) en la UNAM. Sus temas de investigación se enfocan en el desarrollo del concepto de adolescencia en México y Estados Unidos y en la historia de la formación, cultura material e identidades de los adolescentes. Es fundadora y coordinadora del Seminario Interinstitucional de Historia de las Juventudes en el IISUE). Miembro de la Red Nacional de Jóvenes Investigadores sobre Juventud del Seminario de Investigación en Juventud de la UNAM y de la Red de Estudios de Historia de las Infancias en América Latina. Entre sus últimas publicaciones se encuentran "Ezequiel A. Chávez y su Ensayo de psicología de la adolescencia (1928): una versión moderna de los adolescentes mexicanos", Relaciones, Revista de Historia y Sociedad (en imprenta, publicación prevista para la primavera de 2017); su tesis doctoral "La edad difícil. Los adolescentes modernos de la ciudad de México (1876-1934)", México, El Colegio de México, 2015, y “De la Universidad al campo: El establecimiento del servicio médico social en la Universidad Autónoma de México (1934-1940)", Historia Mexicana, núm. 254, octubre-diciembre, 2014.

Recibido: 7 de octubre de 2016

Aceptado: 20 de noviembre de 2016 